\title{
Aquaporins and the respiratory system: advice for a lung investigator
}

\author{
Landon S. King, Søren Nielsen, and Peter Agre \\ ${ }^{1}$ Departments of Biological Chemistry and Medicine, Johns Hopkins University School of Medicine, Baltimore, Maryland 21205, USA \\ ${ }^{2}$ Department of Cell Biology, Institute of Anatomy, University of Aarhus, DK-8000 Aarhus, C, Denmark
}

Address correspondence to: Landon S. King, Department of Medicine, Johns Hopkins University School of Medicine, 600 North Wolfe Street, Blalock 910, Baltimore, Maryland 21287, USA.

Phone: (410) 955-3467; Fax: (410) 955-0036; E-mail: lsking@welch.jhu.edu.

\begin{abstract}
"In summary, there are no small problems. Problems that appear small are large problems that are not understood. Nature is a harmonious mechanism where all parts, including those appearing to play a secondary role, cooperate in the functional whole. No one can predict their importance in the future. All natural arrangements, however capricious they may seem, have a function."

-Santiago Ramón y Cajal, Advice for a young investigator, 1897
\end{abstract}

More than a century after these words were first published, their message is no less poignant. This is particularly true for the readers of the JCI, who find in each issue exciting analyses of mouse knockouts, many of which illustrate the important roles of a wide variety of target proteins. Despite our progress since Cajal's time, it is still worth reminding ourselves of his friendly admonition.

To date, 10 mammalian members of the aquaporin water channel family have been identified, and so far descriptions of 5 aquaporin-null phenotypes have been reported (AQP0, AQP1, AQP2, AQP4, and AQP5) (1-6). This issue of the JCI contains a fascinating description of the apparent role (or lack thereof) played by AQP5 in distal lung (7). In keeping with the complexity of the mammalian lung, multiple aquaporins have been identified at each level of the respiratory tract $(8,9)$. Gas exchange occurs in the distal lung, where most of the alveolar surface is lined by a flattened epithelium consisting of type I pneumocytes surrounding cuboidal type II pneumocytes that secrete surfactant and surfactant proteins, and express the epithelial sodium channel $(\mathrm{ENaC})$. Defects in the genes for surfactant or for the $\alpha$ subunit of $\mathrm{ENaC}$ result in severe pulmonary compromise of the neonatal lung $(10,11)$. AQP5 is found at the apical membrane of type I pneumocytes, but no aquaporin has been identified in the basolateral membrane of these cells, or in type II pneumocytes (Figure 1a) (9). The postnatal expression of AQP5 in the distal lung (12) and the appearance of AQP5 in differentiating alveolar pneumocytes have both been described
(13). Just below the alveolar basement membrane reside capillaries that express constitutively active AQP1 in relatively low concentrations (Figure 1a). In the airways and nasopharynx, the plumbing system appears to be no less complex, with AQP1, AQP3, and AQP4 expressed in 3 distinct but adjacent locations (9). In the submucosal secretory glands, AQP5 is abundant in the apical membranes of glandular acini, whereas basolateral membranes contain other aquaporins (Figure 1b). It was originally predicted that AQP5 would play a major function in glandular secretions (14), and indeed, AQP5deficient mice show a marked reduction in saliva formation (6).

Based upon these complex distributions, it is likely that respiratory tract aquaporins each play unique roles. Unfortunately, unlike the mammalian kidney, for which there is general agreement about transport mechanisms, details of the fluid transport pathways in the respiratory system continue to be the

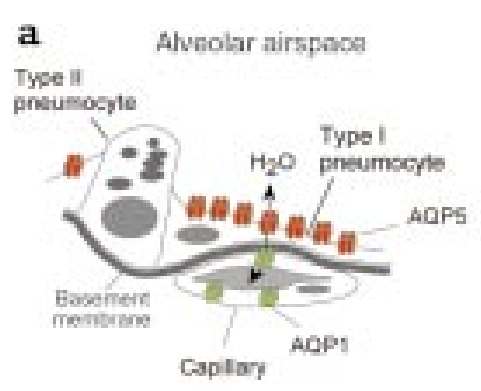

subject of vigorous debate. Therefore, preparation of mice that are homozygous for disruptions in the aquaporins of the respiratory system may provide important information about these interesting proteins, but, as in kidney research, comprehensive and difficult physiological approaches are required. Ma and colleagues (7) measured alveolar permeability in control mice and AQP5-deficient mice using 3 models: (a) generation of an osmotic gradient from the vascular bed to the alveolar airspace, (b) clearance of isosmolar fluid from the alveolar space, and (c) generation of pulmonary edema in response to hydrostatic pressure gradients. In the first set of studies, the authors demonstrated - using both a pleural surface fluorescence technique with an alveolar tracer and a classical gravimetric (or lung weight) method that the alveolar permeability of AQP5null mice is decreased 10 -fold in response to an osmotic gradient. Such a result might have been expected,

\section{b} Submucosal secretory gland

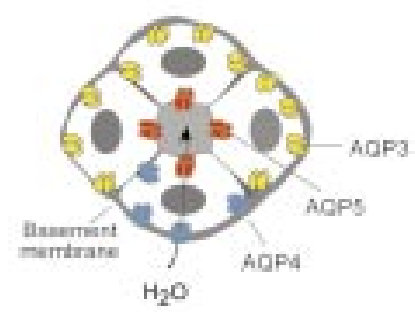

Figure 1

Diagrammatic representation of aquaporin expression in select respiratory tissues. (a) Distal lung showing alveolar airspace, type I pneumocytes containing AQP5 in the apical membrane, type II pneumocytes with no known aquaporins, and underlying capillary containing low levels of AQP1. (b) Submucosal secretory gland acinus containing apical AQP5 and basolateral AQP3 or AQP4. This figure is modified and reproduced with permission (9). 
because AQP5 is abundant in type I pneumocytes (9), and isolated type I pneumocytes are known to have high osmotic water permeability (15). The abundance of AQP5 at this site may facilitate rapid and extensive water absorption during freshwater drowning, in which victims may suffer hemolysis, cardiac shock, and cerebral edema.

In contrast to this clear defect in lung permeability to osmotic gradients, the investigators find that AQP5-deficient mice are indistinguishable from wild type with regard to hydrostatic pulmonary edema or isosmolar fluid transport from the alveolar space. The lack of difference in isosmolar fluid reabsorption may be surprising at first glance, because AQP5 might normally be expected to function as an entryway for transcellular water flow. Drawing on estimates suggesting relatively low fluid absorption rates in the alveolus, the authors therefore propose that AQP5 may be an evolutionary remnant of little physiological importance to type I pneumocytes.

The specific pathway for fluid exit from the alveolar space is as yet undefined, and the requirement for generation of an osmotic driving force might focus attention away from the type I pneumocyte. Despite its apparent lack of involvement in active near-isosmolar water transport in the alveolus, it seems likely that AQP5 may serve other functions, such as generation and maintenance of an aqueous surface layer, or perhaps mediating $\mathrm{CO}_{2}$ exchange, as has been postulated for AQP1 (16). Alternatively, AQP5 might help in regulating type I pneumocyte volume, or in maintaining the attenuated cytoplasm of the type I cell, which contributes to the efficiency of gas exchange.

Whatever the role of this transporter, the data of $\mathrm{Ma}$ and colleagues (7) regarding the transmission of the AQP5-null allele indicates that AQP5 must be very important during the first 5 days of life for these animals. The authors found a marked reduction in both $A Q P 5^{+/}$heterozygotes and $A Q P 5^{-/-}$homozygotes among the offspring of $A Q P 5^{+/-}$matings. The identification of humans lacking functional AQP1 (2) or AQP2 (4) strongly predicts that people with dysfunction of the other aquaporins are still awaiting recognition, although their phenotypes may not always reflect those of their murine distant cousins.

Recent suggestions that investigation of aquaporins in the lung is no longer warranted, inspired by this failure to find a predicted defect in the distal lungs of AQP5-deficient mice, presumes that scientists fully understand the repertoire of functions played by the known aquaporins - probably a premature idea from Cajal's viewpoint. Fortunately, reports such as that by $\mathrm{Ma}$ and colleagues (7) in this issue of the JCI demonstrate the need for more study in this area, not less. The free availability to all scientists of publicly reported studies using transgenic mice (a policy that this and other journals promote) should advance this field by permitting the testing of novel ideas about the roles of these proteins. Moreover, methods are needed to determine transport at distinct locations in the respiratory tract and alveolus, as are high-resolution histochemical studies of aquaporin expression in the respiratory tract under stress. The search for still-unrecognized aquaporins in respiratory tissues should also be intensified, and murine models that allow for conditional expression of aquaporins may be necessary if we are to deepen our understanding of this important class of molecule.
1. Shiels, A., and Bassnett, S. 1996. Mutations in the founder of the MIP gene family underlie cataract development in the mouse. Nat. Genet. 12:212-215.

2. Preston, G.M., Smith, B.L., Zeidel, M.L., Moulds, J.J., and Agre, P. 1994. Mutations in aquaporin-1 in phenotypically normal humans without functional CHIP water channels. Science. 265:1585-1587.

3. Ma, T., et al. 1998. Severely impaired urinary concentrating ability in transgenic mice lacking aquaporin-1 water channels. J. Biol. Chem. 273:4296-4299.

4. Deen, P.M.T., et al. 1994. Requirement of human renal water channel aquaporin-2 for vasopressindependent concentration of urine. Science. 264:92-95.

5. Ma, T., et al. 1997. Generation and phenotype of a transgenic knockout mouse lacking the mercurial-insensitive water channel aquaporin-4. J. Clin. Invest. 100:957-962.

6. Ma, T., et al. 1999. Defective secretion of saliva in transgenic mice lacking aquaporin-5 water channels. J. Biol. Chem. 274:20071-20074.

7. Ma, T., Fukuda, N., Song, Y., Matthay, M.A., and Verkman, A.S. 1999. Lung fluid transport in aquaporin-5 knockout mice. J. Clin. Invest. 105:93-100

8. King, L.S., Nielsen, S., and Agre, P. 1996. Aquaporin-1 water channel protein in lung: ontogeny, steroid-induced expression, and distribution in rat. J. Clin. Invest. 97:2183-2191.

9. Nielsen, S., King, L.S., Christensen, B.M., and Agre, P. 1997. Aquaporins in complex tissues. II. Cellular and subcellular distribution in respiratory tract and glands of rat. Am. J. Physiol. 273:C1549-C1561.

10. Jobe, A. 1984. Respiratory distress syndrome: new therapeutic approaches to a complex pathophysiology. Adv. Pediatr. 30:93-130.

11. Hummler, E., et al. 1996. Early death due to defective neonatal lung liquid clearance in alphaENaC-deficient mice. Nat. Genet. 12:325-328.

12. King, L.S., Nielsen, S., and Agre, P. 1997. Aquaporins in complex tissues. I. Developmental patterns in respiratory tract and glandular tissue of rat. Am. J. Physiol. 273:C1541-C1548.

13. Borok, Z., et al. 1998. Keratinocyte growth factor modulates alveolar epithelial cell phenotype in vitro: expression of aquaporin-5. Am. J. Respir. Cell Mol. Biol. 18:554-561.

14. Raina, S., Preston, G.M., Guggino, W.B., and Agre, P. 1995. Molecular cloning and characterization of an aquaporin cDNA from salivary, lacrimal, and respiratory tissues. J. Biol. Chem. 270:1908-1912.

15. Dobbs, L., et al. 1998. Highly water-permeable type I alveolar epithelial cells confer high water permeability between the airspace and vasculature in rat lung. Proc. Natl. Acad. Sci. USA. 95:2991-2996.

16. Nakhoul, N.L., Davis, B.A., Romero, M.F., and Boron, W.F. 1998. Effect of expressing the water channel aquaporin- 1 on the $\mathrm{CO}_{2}$ permeability of Xenopus oocytes. Am. J. Physiol. 274:C543-C548. 